\title{
Flight Speed Derivation of Helicopter Mobile System
}

\author{
Kazunari Kuwahara \\ KDDI Research, Inc.
}

\author{
Hiroya Tanaka \\ KDDI CORPORATION
}

\author{
Yoshinori Kitatsuji \\ KDDI Research, Inc.
}

\begin{abstract}
This paper derives a maximum flight speed for a helicopter mobile system (HMS) through verifying the communication area of HMS. This prevents the mobile terminal of disaster victims to miss detecting radio wave when the HMS's radio wave reaches them. The HMS is proposed to be used when a big disaster damage occurs and an area where the base stations stop appears. At such a disaster situation, HMS and this clarified maximum flight speed contribute to shorten a search process in rescuing the disaster victims.
\end{abstract}

\section{CCS CONCEPTS}

- Applied computing; • Aerospace;

\section{KEYWORDS}

Helicopter mobile system, Location estimation, Radio wave propagation

\section{ACM Reference Format:}

Kazunari Kuwahara, Hiroya Tanaka, and Yoshinori Kitatsuji. 2021. Flight Speed Derivation of Helicopter Mobile System. In The 2021 9th International Conference on Computer and Communications Management (ICCCM '21), fuly 16-18, 2021, Singapore, Singapore. ACM, New York, NY, USA, 6 pages. https://doi.org/10.1145/3479162.3479192

\section{INTRODUCTION}

When a disaster occurs, there is a situation where it becomes difficult to search disaster victims in non-operated cellular areas, or places where base stations stop their operations. This is a significant issue because the survival rate of disaster victims decreases with increasing the search time. A mobile network operator can recently provide the latest positions where the mobile terminals make communications to rescue agencies utilizing temporary base stations, and this can often support to save lives of disaster victims.

We have been proposing an aerial mobile system which is an aerial vehicle mounting a mobile system enabling mobile terminals in the ground to communicate in its radio wave range. The realized communications by this system are delivering notifications (oneway messaging) and short messages (bi-directional messaging), making calls, and collecting GPS information. We expect that the aerial mobile system can support rescuing people in mountainous distress or victims in disaster situation.

There is an issue in the aerial mobile system. The rescuer (persons to be rescued) should be identified in advance and the aerial

Permission to make digital or hard copies of all or part of this work for personal or classroom use is granted without fee provided that copies are not made or distributed for profit or commercial advantage and that copies bear this notice and the full citation on the first page. Copyrights for components of this work owned by others than ACM must be honored. Abstracting with credit is permitted. To copy otherwise, or republish, to post on servers or to redistribute to lists, requires prior specific permission and/or a fee. Request permissions from permissions@acm.org.

ICCCM '21, July 16-18, 2021, Singapore, Singapore

(c) 2021 Association for Computing Machinery.

ACM ISBN 978-1-4503-9007-1/21/07 ..\$15.00

https://doi.org/10.1145/3479162.3479192 mobile system carries his/her secret key for communications made by his/her mobile terminals. Suppose that helicopter mounting mobile system (called 'HMS' standing for 'helicopter mobile system' hereafter) tries to find an unidentified person. Although the solution can be that the HMS carries all the secret keys of all the users of a mobile network operator, this is difficult due to a significant damage in the case of the information (secret keys) leakage.

HMS has an additional solution: estimating the location of mobile terminals, and which, leverages an initial signal of attach procedure as a beacon. The basic idea is utilizing multiple signals, and the HMS narrows an area, where the mobile terminal exists, with overlaps of radio-wave ranges corresponding to the individual captured signals.

Suppose the helicopter flies over a large area. The high-speed flight is required to shorten the search time. However, the mobile terminal has an interval to scan radio wave from the base station. Therefore, the HMS may miss-detect the mobile terminal when it flies at high-speed. This is a significant issue because the missdetecting result in a delay in finding disaster victims. This paper derives the maximum flight speed, at which the HMS do not missdetect the victim's mobile terminal, through field verification of radio propagations.

The contributions of this research are that: (1) we clarified the process of search activities for disaster victims in the case of utilizing HMS; (2) the bag-style HMS, which can be carried in various helicopters, is developed; and (3) the maximum flight speeds for HMS are derived in order not to miss-detect the mobile terminal on the ground while the HMS flies. These contributions are expected to drastically shorten a search time compared to the conventional search process using the helicopters (e.g., half a day to 30 minutes).

This paper organizes as follows: Section 2 describes the related work and clarifies the needs of our work; Section 3 describe an approach to introduces the mobile system into person-search operation in rescue activities; Section 4 describes field verification of radio propagations for individual medium- and small-sized helicopters and how to derive the maximum flight speed; and Section 5 concludes this paper.

\section{RELATED WORK}

Although there are dedicated communications systems, such as, police, firefighter, and self-defense force (in Japan), they are not for public uses.

In Japan cases, municipalities build a radio communication system for the disaster management [1]. This system is limited to one-way communication (for notification purposes), such as, notifying risks of the upcoming disaster (damage estimation) and/or how to minimize its damage by preparing in advance.

Internationally, the 4G-LTE packet core function is being considered as part of public safety [2], but it is targeted at dedicated terminals. The products actually developed are also considered to be for public safety or military use [3, 4], and the radio waves used are dedicated and cannot be used by general users. 
Multiple small unmanned aerial vehicles (UAVs) can form communication area with a flying ad-hoc network (FANET) [5-7]. This is one of solution applied, instead of cellular communications, for delivering user data of mobile terminal to the public communication network (i.e., the Internet). Additionally, multiple UAVs can cover a large area for mobile terminals communications. An issue is a long flight of individual UAVs when this solution is applied to mountain region, such as, where originally cellular system is not built. Covering a large area inherently requires long-haul flights, and the actual service delivery time would be even shorter.

SARDO [8] is the first cellular-based search system for the disaster victims or persons in mountainous distress. This technology uses time of flight (ToF) for radio wave and the trilateration, and can estimate the position of mobile terminal. Although this solution can estimate highly accurate position of mobile terminal with 50-meter error at minimum, the applied range of radio wave is small, e.g., around $500 \mathrm{~m}$. This also has the problem of short flight time due to the characteristics of UAV.

In the scenario of this paper, a single HMS searches a person of the mountain distress in an area of $20-200 \mathrm{~km}^{2}$ from the sky. Ground rescue teams (10 to 100 persons) simultaneously enters from multiple entrance points on the ground. A key to shorten the rescue time is how quickly the HMS detects the first signal of victim's mobile terminal. This enables the ground rescue team to rush ahead for the notified position where the HMS has detected the signal. While this, the HMS narrows the location of the victim with multiple signals of his/her mobile terminal. We assume that the HMS in this scenario narrows victim's location and but not collects the his/her GPS information, and that the fire or police departments operate their own HMS without the help (the secret key) from the mobile network operator.

\section{DESIGNING HELICOPTER MOBILE SYSTEM}

\subsection{Process of rescue activities}

Generally, a rescue activity starts from a rescue solicitation from relatives of the victim, in the mountain-distress case. And this solicitation usually comes in the (late) evening of the day when the rescued person should have returned home. At this day, the rescue teams (helicopter crews and ground members) make a rescue plan including specifying the search area, which flight and ground paths are taken by the individual members, and so forth. The rescue activity starts in the early morning (just after the sun rises) at the next day.

In the conventional rescue activities (where disaster prevention helicopters are used), the helicopter takes time to find the victim. This is because the helicopter flies looking for the victim at very low altitudes (10 meters at lowest, if they can) along mountain trails. The helicopter uses downwash [9] which rocks the vegetation to search the rescuer. This makes the helicopter slowdown in its flight.

The ground members also search the rescuer along the mountain roads. The ground members climb while confirming the nonexistence of rescuer under the slope of the mountain road, as the rescuer may have slipped off the mountain road. This takes a long time.

After the helicopter or ground member finds the victim, this is notified to all the members, and rescuing starts. If the helicopter

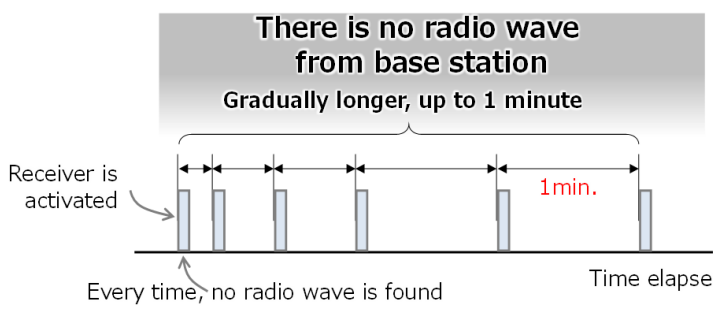

Figure 1: Intervals of mobile terminal for scanning radio wave from base station.

cannot hover directly above the rescuer, the ground members carry the rescuer to a place where the helicopter easily pulls up the rescuer.

In such a rescue activity, the search process takes around 1 to 4 hours.

\subsection{Required search operation with helicopter mobile system}

In the rescue activity, we propose to utilize the communication with rescuer's mobile terminal. The search process for undefined rescuer would be followings:

- the HMS detects a signal of rescuer's mobile terminal;

- the HMS notifies all the members the first position;

- the ground rescue members directly rush ahead for the notified position;

- the helicopter continues to capture signals from victim's mobile terminal in an attempt to narrow the area where the victim is (the helicopter may pull down the crew to the narrowed area in order to search the victim);

- the ground members arrive the narrowed area to search the rescuer; and

- eventually, they find the rescuer.

In these steps, it is assumed that the rescuer terns on the mobile terminal upon hearing the sound of a helicopter flying if it had been turned off. When the rescuer is identified in advance and the HMS carries his/her secret key, GPS information of his/her mobile terminal can be collected, which eliminate the attempt to narrow the area from step 4 of the above search process.

In general, the mobile terminal has its interval to scan radio wave from base station, as illustrated in Figure 1. First, the rescuer's mobile terminal has original found that there was no radio wave from base station. This leads the scanning interval increasing. In the case of the $4 \mathrm{G}$ LTE mobile terminals, the interval increases up to 1 minute. This maximum interval is the reason to limit the flight speed of HMS. The radio wave from it must keep deliver its radio wave more than 1 minutes even if HMS flies fast. The actual flight speed is derived in Section 4.1.

\subsection{Mobile system mounted in helicopter}

Regarding the proposed HMS, a crew boards the helicopter by carrying a bag with a built-in mobile system including an antenna, base station, mobile core, location service, IMS (IP Multimedia Subsystem), cell broadcast center, and earthquake tsunami warning 


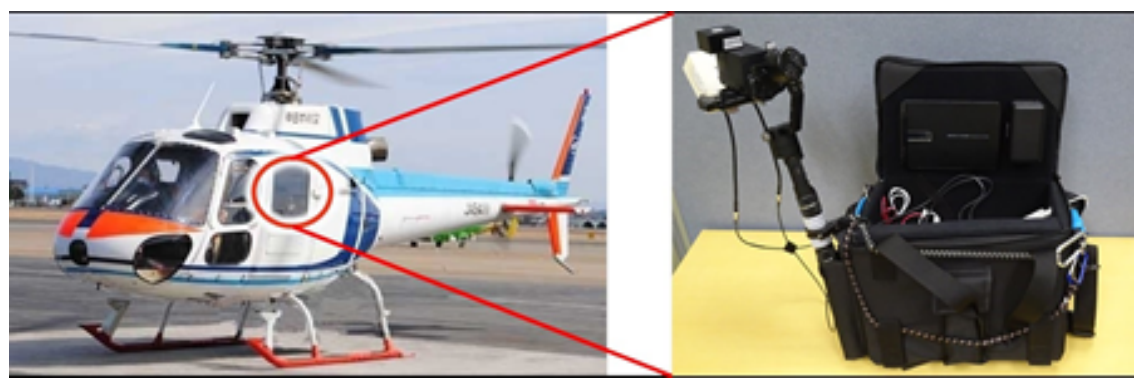

Figure 2: A helicopter mounting a prototype of bag-style mobile system.

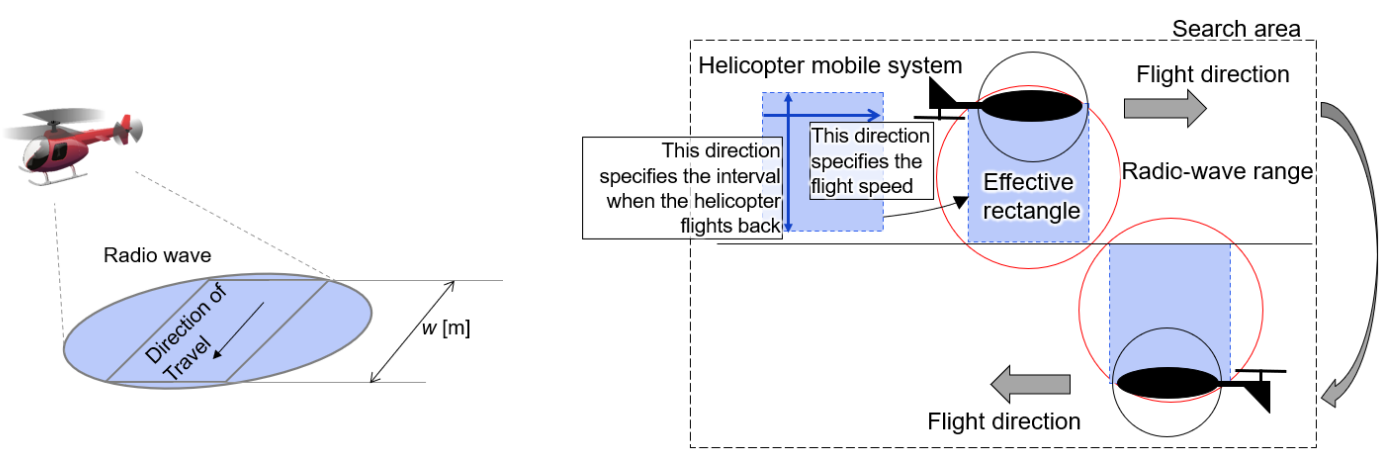

Figure 3: Effective rectangle in the range of radio wave (left-hand side) and flight path with the effective rectangle.

system (Figure 2). The bag is tied to the window seat of a helicopter to point the antenna tilted diagonally toward the ground through the window on one side of the helicopter. The antenna is attached on the three-axis gimbal with the stabilizing capability which control the direction of the antenna according to the change of helicopter's pose (i.e., yaw, pitch, and roll rotation). The reason we take the bag style is that we would like to bring this prototype to the various helicopters used for the rescue activities as the HMS for the field trials and demonstrations.

When this HMS flies, a crew operates this bag through a dedicated controlling terminal showing the number of mobile terminals for which signals are captured, the detail position via GPS information, the communication status (reachability, call and messaging) of the mobile terminals if the secret key is available. This crew can also understand which region in the search area has been already searched through this control terminal. The crew indicates the pilot which direction the helicopter ahead to find the rescuer's mobile terminal.

In the case of implementing a dedicated helicopter mounting the mobile system, the antenna should be attached directly to the bottom of the fuselage, and the mobile system should be installed without blocking any seat. These remain as future work.

\section{DERIVATION OF FLIGHT SPEED FOR HELICOPTER MOBILE SYSTEM}

As described in the section 3.2, HMS should set a limit on flight speed so that it can communication with the mobile terminals without omissions. This subsection derives the maximum flight speed of helicopter.

Figure 3 left-hand side shows the effective rectangle, in the range of radio wave, in which signals cannot be missed the detection by HMS. In the case where the flight speed increases, the effective rectangle gradually becomes elongated in the direction of helicopter travel ( $w[\mathrm{~m}]$ can be diameter of the circle at maximum). Conversely, as the flight speed slows down, the rectangle can gradually widen.

The HMS should fly as filling the search area via the effective rectangle (Figure 3 right-hand side). Therefore, the maximum size of the effective rectangle results in minimizing the time of search process. At this time, the helicopter should take more than a period of the interval of the signal scanning at the mobile terminal: $i$ [sec] (up to 60 seconds) to fly a distance of $w$. Therefore, the flight speed should be less than $w / i[\mathrm{~m} / \mathrm{sec}]$.

\subsection{Experimental environment and system setting}

To derive the actual helicopter flight speed, we had a field experiment to clarify the range of the radio wave. The experimental environment and system setting are specified in Table 1 . We used two different sizes of helicopters, and measured signal strengths at various distances from each helicopter. The mobile system is implemented for 4G-LTE mobile terminals.

Figure 4 shows AgustaWestland AW139 (the medium-sized helicopter) used for rescuing people in mountainous distress or victims in disaster situations. Figure 5 shows the antenna and directivity of radio wave which is emitted from the antenna. The antenna is 
Table 1: Experimental environment and system settings.

\begin{tabular}{ll}
\hline Parameters for setting & Values \\
\hline Helicopter & AgustaWestland AW139 and AW109 \\
& $($ Medium- and small-sized helicopters, respectively) \\
Helicopter altitude & $300 \mathrm{~m}$ \\
Directions from antenna direction & $-90^{\circ},-60^{\circ},-30^{\circ}, 0^{\circ}, 30^{\circ}, 60^{\circ}$ and $90^{\circ}$ \\
Distance from helicopter & $1,2,3$ and $4 \mathrm{~km}$ \\
Radio output & $50 \mathrm{~mW} \times 2$ \\
Radio communication method & OFDM $-\mathrm{FDD}$ \\
Center Frequency & $2125 \mathrm{MHz}$ \\
Occupied Bandwidth & $10 \mathrm{MHz}$ \\
Feed line loss & $1 \mathrm{~dB}$ \\
Antenna gain & $5 \mathrm{dBi}$ \\
Radio polarization & Circularly polarized wave \\
\hline
\end{tabular}
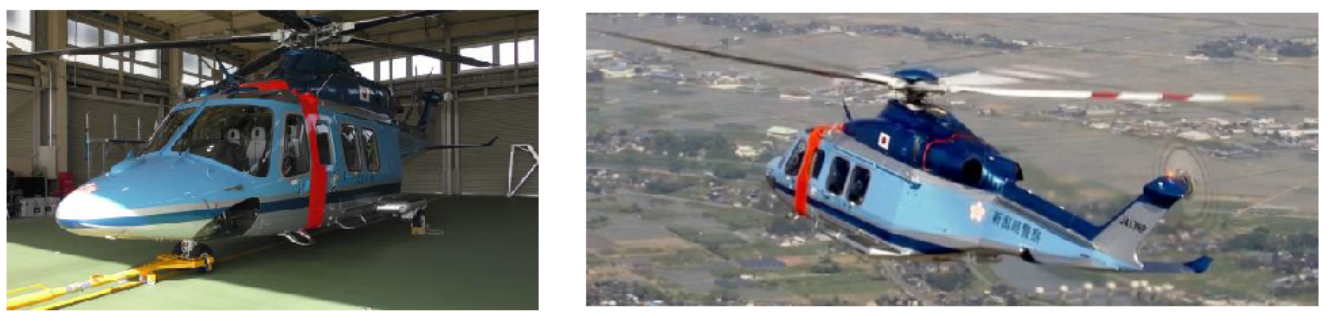

Figure 4: AgustaWestland AW139 (named 'Koshikaze'), a medium-sized helicopter, used in the field experiment.

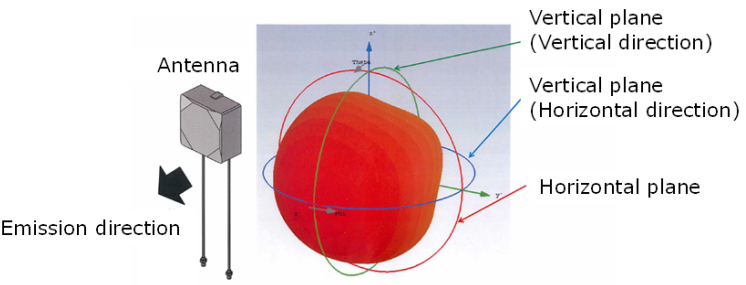

Figure 5: Antenna and directivity of radio wave emitted from the antenna.

circular polarization, whose radiation patterns of vertical plane directivity are shown in Figure 6 (two on the left are horizontal direction and two on the right are vertical direction).

\subsection{Radio wave propagations and Flight speeds}

We investigated shapes of the effective radio wave areas in cases of the medium- and small-sized helicopters. The effective radio wave area is an area where the mobile terminal can detect the radio wave from HMS and progresses the signal processing when the helicopter is hovering. During the measurements, the helicopter kept hovering in order to keep the constant pose (i.e., pitch and roll angles were constant).

Figure 7 left-hand side shows the signal strength for each pair of distances $(0-3 \mathrm{~km})$ and angles $\left(-90^{\circ}-+90^{\circ}\right)$ in the case of the medium-sized helicopter. $-90^{\circ}$ of direction indicates the head of helicopter (traveling direction). All the measured signal strength had the line of sight.

This figure also shows the radio wave area boundary $(-125 \mathrm{dBm})$ as the effective radio wave area. The value: $-125 \mathrm{dBm}$ is the minimum signal strength at which the mobile terminal progress signals. This value was preliminary measured before this experiment was conducted. To be precise, this boundary line was plotted at points of $-125 \mathrm{dBm}$ by Euclidean distance calculation from the values of signal strengths at the measurement points of $0-3 \mathrm{~km}$ and $-90^{\circ}-$ $+90^{\circ}$.

Figure 7 right-hand side shows the largest rectangle contained in the effective radio wave area (left-hand side). To discover the maximum size of rectangle, each vertex of rectangle is attached to the edge of the effective radio wave area to slide (each color of plots corresponds to each vertex). When sliding the left-top (green) vertex, it has reached the maximum area in all the vertexes. Therefore, the maximum flight speed is derived by the length of the rectangle in the travelling direction (y axis), that is, $2.08 \mathrm{~km} \times 60$ $\min =124.4[\mathrm{~km} / \mathrm{h}]$. If the helicopter exceeds this speed, the HMS may not detect the signal from mobile terminal on the ground.

Similarly, for small-sized helicopter, the maximum flight speed was $159.4 \mathrm{~km} / \mathrm{h}\left(=2.66 \mathrm{~km}{ }^{*} 60 \mathrm{~min}\right)$, as illustrated in Figure 8 . The effective radio wave area becomes larger than the case of middlesized helicopter. Therefore, the effective rectangle also becomes larger, and the maximum flight speed increases. 


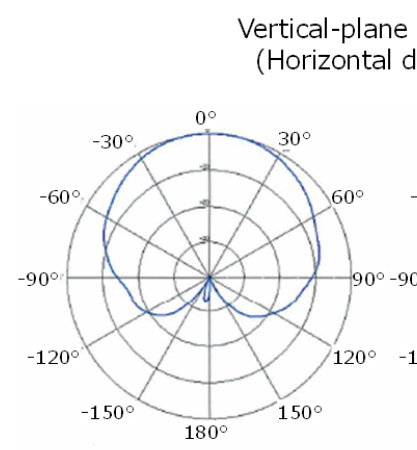

Left-handed circularly polarized wave

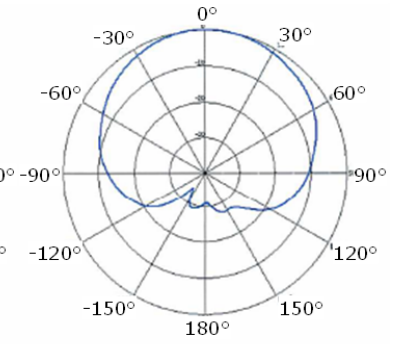

Right-handed circularly polarized wave
Vertical-plane directivity

(Vertical direction)

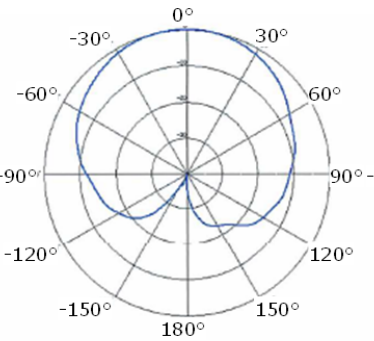

Left-handed circularly polarized wave

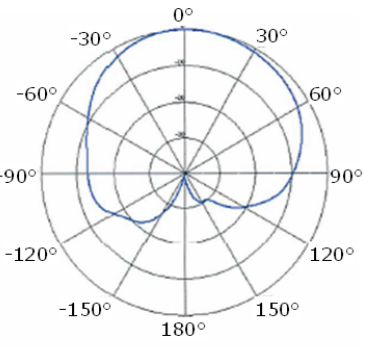

Right-handed circularly polarized wave

Figure 6: Vertical-plane directives for horizontal and vertical directions.
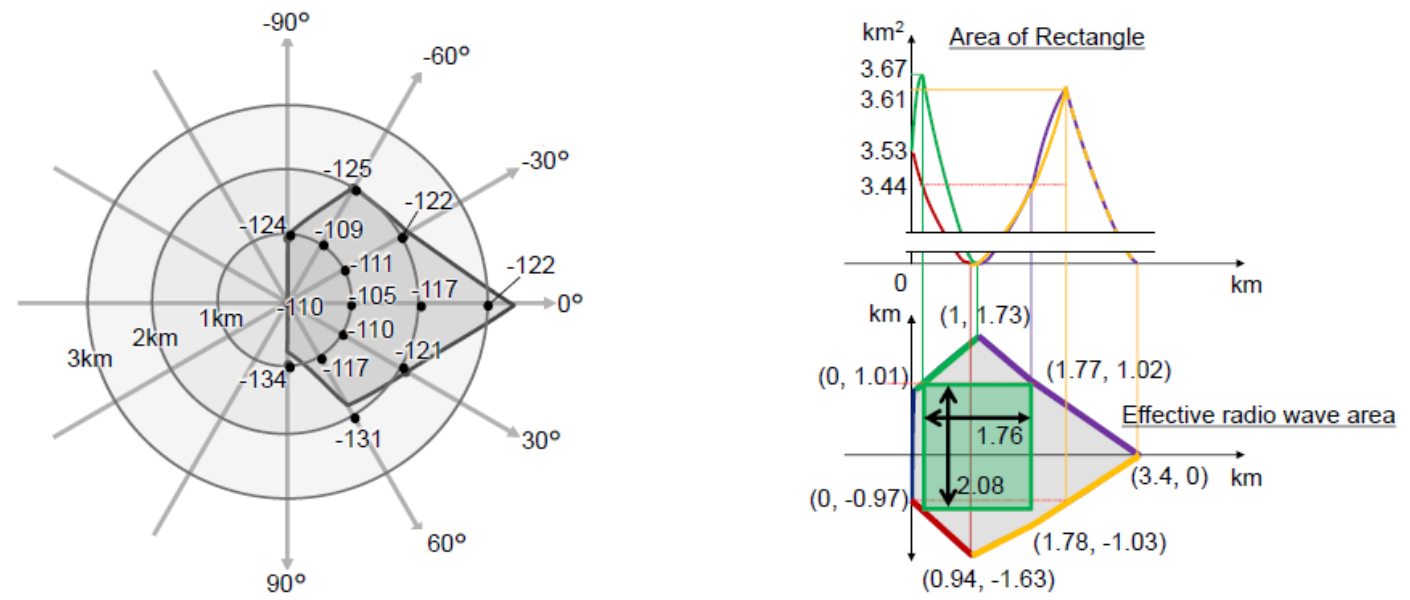

Figure 7: The effective radio wave area (left-hand side) and maximum effective rectangle (right-hand side) for the mediumsized helicopter.
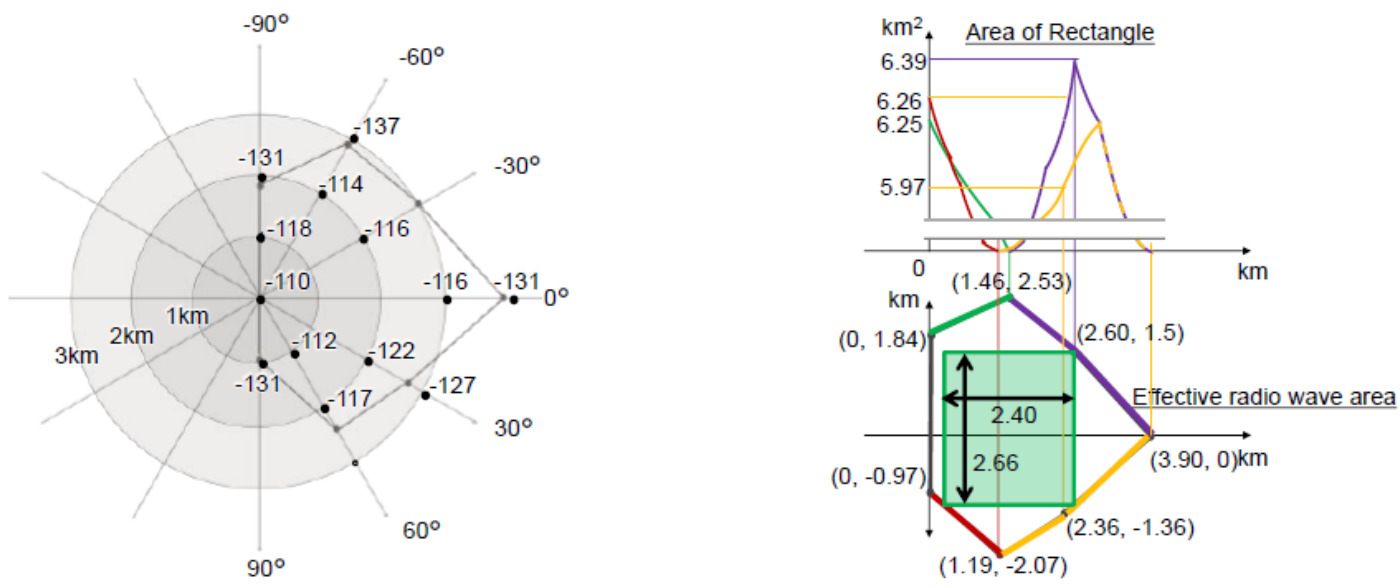

Figure 8: The effective radio wave area (left-hand side) and maximum effective rectangle (right-hand side) for the small-sized helicopter. 


\subsection{Discussion on the applying the real search activities}

The derived maximum flight speed works in a limited situation conditioned by Table 1 . On the other hand, depending on the conditions: flight altitudes; body shapes of helicopter; materials of windows and so forth, the maximum flight speed varies. In terms of the search operation in rescue activities, the rescue teams generally have designated activity areas, and flight plans and/or paths depend on each of these areas.

Currently, we take up multiple combinations of conditions and perform experiments corresponding to them. The important conditions are flight altitudes, helicopter shape, window materials, and radio outputs. Their variations easily influence the shape of effective radio wave areas, and rectangles.

One of difficult things is that the flight altitude (precisely, distances between ground surface and helicopter) varies in a single flight. Because we will have a limited number of field verifications against a huge amount of variations from the combinations of the conditions, a maximum flight speed for the lowest flight altitude (bringing the lowest flight speed) should be taken for the search operation as a minimum preparation. If the search operation can utilize pilot skills, keeping a certain distance from ground surface when the helicopter flies allows the HMS to take the higher maximum flight speed in the search operation. In order to take a simple flight method, let the build-in mobile system track the distances from the ground surface by sequential comparisons to altitude map for the flight area, and sequentially indicate the flight speed in a flight. this approach should have a method to be able to estimate the shapes of effective radio wave areas.

\section{CONCLUSION}

This paper showed a way of derivation of the maximum flight speed for the helicopter mobile system (HMS). The maximum flight speed is for preventing the mobile terminal in the ground to miss detecting the radio wave from the HMS. It is expected to shorten the search and rescue activities and save disaster victim's life by utilizing our proposal. This derivation was conducted through the field experiments clarifying the effective radio wave areas in which the 4G-LTE mobile terminals could progress signal processing with the HMS while the HMS flew over the mobile terminals. Comparing between medium- and small-sized helicopters, the small-sized one showed a larger effective radio wave area, and resulted in a higher (faster) maximum flight speed. (159.4 km/h and $124.4 \mathrm{~km} / \mathrm{h}$ for medium- and small-sized helicopters, respectively).

The effective radio wave area varies depending on various conditions, such as, flight altitudes, airframe shape of helicopter, window materials. Many of these parameters are preliminarily fixed, and therefore, the effective radio wave areas can be derived via preliminary field experiments. However, in order to correspond to the flight altitude variation, it is necessary to devise a method to automatically estimate the effective radio wave areas (their shapes and sizes) during the flight. This remains as one of future works.

\section{ACKNOWLEDGMENTS}

Apart of this work was supported by "Investigation and examination of disaster countermeasure wireless communication system for mobile terminals" project funded by Japan Ministry of Internal affairs and Communications in 2015 - 2017, "Cross-ministerial Strategic Innovation Promotion Program - Theme I: development of integrated system for evacuation/emergency activity support" project funded by Japan Cabinet Office in 2018 and 2019, and Niigata Prefectural Police Aviation Unit in 2020.

\section{REFERENCES}

[1] Y. Goto, T. Mikami, and I. Nakabayashi. 2012. Fact-Finding about the Evacuation from the Unexpectedly Large Tsunami of March 11, 2011 in East Japan. In Proceedings of 15th World Conference on Earthquake Engineering 2012 (15WCEE). Curran Associates, Inc., 57 Morehouse Lane Red Hook, NY 12571, ISBN: 978-63439-651-6.

[2] 3GPP. 2015. Study on architecture enhancements to support isolated Evolved Universal Terrestrial Radio Access Network (E-UTRAN) operation for public safety. TR 23.797, ver. 13.0.0, Jun. 2015.

[3] Motorola. 2016. Portable Public Safety LTE Makes Real-Time Information Available Anywhere, Anytime. In Motorola Solutions, White Paper, Aug. 2016.

[4] Radisys. 2016. Multi-Radio Network-in-a-box. Octasic and Quortus. http://www.octasic.com/radisys-octasic-and-quortus-to-deliver-a-multiradio-network-in-a-box-for-defense-and-public-safety/.

[5] E.Yanmaz, S. Yahyanejad, B. Rinner, H. Hellwagner, and C. Bettstetter. 2018. Drone networks: Communications, coordination, and sensing. ELSVIER, Ad Hoc Networks, Vol. 68, January 2018, pp. 1-15 from https://www.sciencedirect.com/science/ article/abs/pii/S1570870517301671?via\%3Dihub

[6] L. Ye, Y. Zhang, Y. Li, and S. Han. 2020. A Dynamic Cluster Head Selecting Algorithm for UAV Ad Hoc Networks. Int. Wirel. Commun. Mob Comput.(IWCMC). Limassol, Cyprus. Jun 2020, pp. 225-228. https://ieeexplore.ieee.org/abstract/ document $/ 9148458$

[7] M. Deruyck, J. Wyckmans, L. Martens, and W. Joseph. 2016. Emergency adhoc networks by using drone mounted based stations for a disaster scenario. Proceedings of IEEE WiMob, New York, USA. October 2016, pp. 1-7. https: //ieeexplore.ieee.org/abstract/document/7763173

[8] A. Albanese, V. Sciancalepore, X. Costa-Perez. 2021. SARDO: An Automated Searchand-Rescue Drone-based Solution for Victims Localization. IEEE Transaction on Mobile Computing (Early Access). https://www.computer.org/csdl/journal/tm/ 5555/01/09321707/1qmbiP9aO1q

[9] F. B. Yang, X. Y. Xue, L. Zhang, Z. Sun. 2017. Numerical simulation and experimental verification on downwash air flow of six-rotor agricultural unmanned aerial vehicle in hover. Int J Agric \& Biol Eng, 2017; 10(4): pp. 41-53. 